\title{
Viviendo con poco dinero: Un estudio de pobreza rural
}

\author{
Living with Little Money: A Study on Rural Poverty \\ Doris Fernández Carvajal
}

Recibido: 14/08/2017 • Corregido: 23/05/2018 • Aprobado: 11/06/2018

\section{Resumen}

Este artículo es producto de un trabajo de investigación denominado Estrategias de sobrevivencia de hogares pobres desde la perspectiva de género, realizado en el Instituto de Estudios de la Mujer, de la Universidad Nacional, en Costa Rica, durante los años 2014 y 2015 . Este presenta una especie de "radiografía" sobre cómo estaban viviendo la condición de pobreza, un grupo de familias rurales, residentes en las comunidades de Acosta, Isla de Chira y Palenque Margarita, de las provincias de San José, Puntarenas y Alajuela, respectivamente. El mismo enfatiza la dimensión económica de las familias, particularmente lo relativo a los ingresos y gastos, mostrando cómo se generan los recursos económicos, qué destino se les da, quién(es) los aportan, cómo resuelven lo relativo a las necesidades vitales de alimentación, vivienda, salud, recreación y vestimenta y cómo impacta esto en su calidad de vida. La información se recopiló a través de un cuestionario que fue aplicado a cincuenta hogares nucleares y mono parentales a cargo de una mujer. Uno de los hallazgos más relevantes de este estudio, es que demuestra la efectividad metodológica del estudio de la pobreza desde la perspectiva multidimensional. En el caso particular de estos hogares rurales pobres, si bien presentan bajos ingresos, al mismo

1 Master en estudios de la mujer, Docente-investigadora del Instituto Estudios de la Mujer (IEM) de la Universidad Nacional,Heredia, Costa Rica. doris.fernandez.carvajal@una.cr. 
tiempo disponen de una serie de aspectos que contribuyen a mejorar su calidad de vida y que generan sentimientos de inclusión social, dentro de los que se pueden mencionar: el disponer de una alimentación básica, tener acceso a servicios de salud y la tenencia de vivienda propia.

Palabras clave: pobreza; bajos ingresos; hogares pobres; género; Costa Rica.

\section{Abstract:}

This article is based on a research study entitled "Survival Strategies in Poor Households from a Gender Perspective," conducted at the Women Studies Institute from the National University of Costa Rica between 2014 and 2015. This study provides an "X-ray" examination on how poverty was faced by a group of rural families residing in the communities of Acosta, Chira Island and Palenque Margarita in the provinces of San José, Puntarenas and Alajuela, respectively. The paper emphasizes the economic dimension of the families particularly in terms of income and expenses, showing how their economic resources are generated, what they are used for, who contributes to them, how they are used to meet the families" vital needs such as food, housing, healthcare, recreation, and clothing, and how all this impacts the families" quality of life. The information was collected through a questionnaire applied to fifty nuclear homes headed by a single mother. One of the most relevant findings of this study is that it validates the methodological effectiveness of a study on poverty from a multidimensional perspective. In the case of the poor rural homes analyzed, although they have low income, they also have several aspects that help them improve their quality of life and generate feelings of social inclusion, such as having access to a basic diet and healthcare and owning their own house.

Keywords: poverty; low income; poor households; gender; Costa Rica.

\section{INTRODUCCIÓN}

La información para la elaboración de este artículo fue tomada de la investigación denominada, "Estrategias de sobrevivencia de hogares pobres 
desde la perspectiva de género", realizada en el Instituto de Estudios de la Mujer de la Universidad Nacional, Costa Rica durante los años 2014-2015. Esta se desarrolló en tres comunidades rurales costarricenses: Acosta: comunidad rural agrícola ubicada en la provincia de San José; la Isla de Chira: comunidad rural que vive de la pesca artesanal perteneciente a la provincia de Puntarenas y Palenque Margarita: comunidad indígena Maleku, en San Rafael de Guatuso, Alajuela. Con esta escogencia se buscó que las tres comunidades tuvieran características socioeconómicas y culturales diferentes y que permitieran algún grado de representatividad general de la diversidad de comunidades rurales en condición de pobreza en el país. Todo ello con la finalidad de poder explorar factores y causalidades que condicionan la calidad de vida de las personas que habitan en este tipo de comunidades. El abordaje de la pobreza desde la perspectiva multidimensional muestra como estos hogares en condición de pobreza, por bajos ingresos, disponen de una serie de aspectos de su entorno, así como el apoyo de políticas sociales por parte del Estado que logran mejorar su calidad de vida.

Según el Índice de Desarrollo Humano Cantonal (IDHC) del año 2013, los cantones de Acosta, Central de Puntarenas y Guatuso presentan altos porcentajes de pobreza, del $13 \%, 18 \%$ y $21 \%$ respectivamente, también, muestran bajo nivel en desarrollo humano, que las ubican en las posiciones 44,31 y 76 a nivel de los 81 cantones del país. (Castro, 2015).

De acuerdo al Índice de Desarrollo Social (IDS)2, en una escala de 1 a 100 , con este último valor como el estado óptimo o deseable, esas comunidades también exhiben una baja posición, pues se ubican en el lugar 37 , 39 y 23 respectivamente. (Ministerio de Planificación Nacional y Política Económica, 2013).

En Costa Rica, la pobreza afecta a uno de cada cinco hogares, según la Encuesta Nacional de Hogares (ENAHO) aplicada por el Instituto Nacional de Estadística y Censos (INEC) del año 2015, el porcentaje de los hogares en esa condición era del $21.7 \%$ y en pobreza extrema del $7.2 \%$. Por zona geográfica, históricamente la pobreza se ha concentrado mayoritariamente en la zona rural. Para ese mismo año, la pobreza rural afectaba a un $27.9 \%$ de los hogares, mientras que en las urbanas era del $19.5 \%$.

2 El Índice de Desarrollo Social, busca mostrar las posibilidades de acceso y disfrute de que tienen las personas en cuanto a derechos bási-cos en cuatro dimensiones: económica, electoral, salud y educativa. (Ministerio de Planificación Nacional y Política Económica, 2013). 


\section{REFERENTE TEÓRICO}

En esta investigación se aborda el estudio de la pobreza de manera cualitativa, tomando como referencia elementos teóricos y metodológicos de ella desde la perspectiva de género y multidimensional. El uso de métodos cualitativos para analizar la pobreza han venido cobrando mayor importancia, pues se han convertido en "alternativas valiosas para visibilizar desigualdades entre hombres y mujeres respecto de los procesos de adopción de decisiones y distribución de recursos en el hogar, lo que contribuye a abrir la caja negra del hogar y a comprender mejor su funcionamiento". (CEPAL-UNIFEM, 2004, p.30).

La aplicación de la categoría género al estudio de la pobreza pone en evidencia que el mismo hay que entenderlo como un constructo social, dinámico y diverso, que abarca a las mujeres tanto como a los hombres, de ahí que el análisis sea relacional, es decir, obliga a prestar atención no solo a los roles que ejecutan hombres y mujeres, sino que también a la relación que se establece entre ambos. La autora Chant (2003), hace referencia a que:

La pobreza no siempre se ha analizado desde una perspectiva de género. Antes que las feministas contribuyeran en su análisis, se consideraba que la población pobre estaba íntegramente conformada por hombres o bien se daba por sentado que las necesidades e intereses de las mujeres eran idénticos a las de los hombres jefes de hogar, y por ende podían supeditarse a ellos. (p. 10).

Por eso se ha afirmado que la pobreza afecta de manera diferente a mujeres y a hombres. La división sexual del trabajo que asigna la realización de las labores domésticas a las féminas es un factor estructural determinante en la pobreza de las mujeres debida al género. Según Bravo (1998):

La división sexual del trabajo asignada socialmente a las personas de acuerdo a su sexo, no solo tiene efectos materiales concretos en el tipo de trabajos en que se responsabiliza a cada género, sino también un importante efecto en la construcción de identidades de género. (p.61) 
La división del trabajo por sexos está en la base de la desigualdad de oportunidades que tienen las personas de distinto sexo para acceder a los recursos materiales y sociales, propiedad, trabajo remunerado, educación, capacitación, así como a participar en la toma de las principales decisiones políticas, económicas y sociales que forman parte del funcionamiento de una sociedad.

La inclusión de la perspectiva de género ha contribuido también a hacer visible la pluralidad en la composición de los hogares, ya que rompe con el mito de la prevalencia de la unidad doméstica, naturalizada e idealizada como integrada por un esposo, una mujer y unos(as) hijos(as), para visibilizar otras modalidades de hogar, por ejemplo, los que tienen jefatura femenina, haciendo ver que estos están en condición de mayor desventaja en relación con aquellos que están a cargo de un hombre.

Desde hace algunos años se han venido planteando otras alternativas para la medición de la pobreza, una de ellas, es la pobreza multidimensional. Esta considera que el fenómeno de la pobreza es mucho más amplio que la sola falta de ingresos y que tanto esta como las condiciones de vida que experimentan las personas y los hogares no pueden ser medidas a partir de un solo indicador, ya que invisibiliza otros aspectos que inciden en la calidad de vida de las personas. Este método tampoco permite evidenciar el impacto que puedan tener las políticas públicas en diversos ámbitos del bienestar de las personas.

Por eso Kabeer (1998) señala que el análisis de la pobreza multidimensional, tiene un enfoque más holístico, ya que pasa de ver la pobreza de una forma estática, concentrada en los ingresos para reconocer otros aspectos como los económicos, sociales y culturales. En este sentido reconoce que la pobreza es dinámica y multidimensional. Al respecto Kabeer (1998) señala que:

La "Línea de pobreza" define a los pobres como los que no tienen la capacidad de cubrir sus necesidades básicas en calorías, pero una definición más incluyente de necesidad básica abarcaría por lo tanto niveles cultural definidos de bienestar físico (salud, vivienda, vestido, higiene) y si el concepto se amplía podría hasta cubrir los aspectos más intangibles de la privación, tales como la carencia de poder, dependencia y aislamiento. (p.151) 
Este abordaje multidimensional de la pobreza, está inspirado en el economista Amartya Sen, quien la define como "una privación inaceptable de libertades sustantivas de los individuos o como una condición de vida que limita las capacidades de los individuos para vivir la clase de vida que valoran". (Sen, 1999, p.133)

Alkire y Foster (2010), proponentes del Índice de Pobreza Multidimensional (IPM), señalan que el ingreso, no es el único indicador de bienestar, sino que debe ser complementado con otras variables, tales como la salud, la educación y el nivel de vida. Siguiendo a estos autores, la dimensión de la salud incluye la mortalidad infantil y la nutrición; en la educación, es importante cuantificar los años de estudio y el número de niños(as) escolarizados; en el nivel de vida debe considerarse el acceso a electricidad, agua potable, saneamiento, servicio sanitario, energía para cocinar, tipo de piso de la vivienda y tenencia de bienes como televisión, radio, y otros enseres.

La medición de pobreza basada solamente en los recursos,

...no brinda información acerca de las cosas que las personas pueden hacer, o hacen efectivamente, con esos medios. De este modo, las capacidades indicarían las posibilidades o los grados de libertad que las personas tienen para alcanzar determinados funcionamientos, como estar bien nutrido, obtener empleo y educación, o vivir sin sentimientos de humillación ni vergüenza. En esta lógica, la pobreza es la incapacidad de lograr ciertos funcionamientos básicos. (Cepal, 2013, p. 4).

\section{METODOLOGÍA}

Para efectos de este trabajo, la pobreza se determinó a partir de la Línea de Pobreza Rural del año 2014 , que era de $\mathbb{\$} 82.116$ por persona $(\$ 150)$ y la pobreza extrema en $\$ 40.134(\$ 73) .^{3}$

Se utilizó la categoría de hogar dada por el Instituto Nacional de Estadística y Censos, que lo define como "a la persona o conjunto de personas, con o sin vínculo familiar que ocupan en común una vivienda principal o parte de ella y que consumen y comparten alimento con cargo a un mismo

3 Se construyó un promedio tomando como base el dato de la Línea de Pobreza de los meses de marzo a setiembre del 2014. 
presupuesto". (INEC, 2011, p.30) Es importante aclarar que en el texto se hace uso de hogar y familia como si fueran sinónimos, con la finalidad de evitar la repetición y darle más fluidez al mismo.

La investigación incluyó a un total cincuenta hogares, veinte en Acosta y quince en Isla de Chira y Palenque Margarita. De estos, $64 \%$ eran nucleares y $36 \%$ mono parentales a cargo de una mujer. Se eligieron de acuerdo a un criterio visual, focalizando las características físicas de las viviendas, como fachadas despintadas, deterioro en paredes, techos, canoas, ventanas con vidrios quebrados, que al estar en una comunidad de bajo desarrollo socioeconómico, donde predomina pobreza, la probabilidad de encontrarse familias en esta condición fue alta.

La información se recogió a través de un cuestionario con preguntas cerradas y abiertas, ordenado por apartados: ingresos, gastos, vivienda, educación y salud. Fue contestado por las mujeres mediante una visita que realizó la investigadora a sus casas de habitación. La decisión de considerar a las mujeres como informantes, tuvo que ver con el hecho de que son ellas quienes por condición genérica, por lo general, disponen de la información de lo que acontece en la dinámica del hogar.

\section{Algunas características socio demográficas de los hogares}

A fin de tener una mayor claridad de la población estudiada se considera necesario presentar algunos datos que permiten perfilar algunas de las principales características de corte socio demográfico que fueron obtenidas en el proceso de investigación.

Un primer elemento a tomar en cuenta es el hecho de que la totalidad de las familias del estudio son costarricenses, originarias de la zona geográfica y que prácticamente han vivido en el lugar toda su vida, por lo que se puede inferir un alto nivel de arraigo y cohesión social entre ellas.

La mayoría de las mujeres tenían un estado conyugal de casadas y en unión libre, con veinte años de convivencia en promedio. En relación con la edad, las mayores se encontraron en Acosta con 50 años en promedio, las de Isla de Chira y Palenque Margarita, tenían un poco menos edad, 38 y 35 años respectivamente. La edad de los hombres sobrepasa la edad de 
las mujeres, con promedios de 55 años para Acosta, 45 y 38 para las otras dos comunidades.

Los hogares más numerosos se encontraron en Isla de Chira con cuatro hijos(as) en promedio, mientras que en las otras dos comunidades era de tres. La mayor parte de ellos(as) eran menores de dieciocho años, esto muestra el alto nivel de dependencia que tienen con respecto al cuidado, alimentación, educación y salud por parte de sus padres y madres. Estas familias sobrepasan el promedio nacional que es de 1.76 hijos por cada mujer para el año 2015. (INEC, 2016, p.15)

Si bien predomina una baja escolaridad en ambos sexos, en los hombres se encontraron cifras que muestran una situación peor en relación con las mujeres. Un $73 \%$ logró completar la primaria y una cuarta parte presentaba primaria incompleta y analfabetismo. Estos mismos datos en las mujeres, eran de un $69 \%$ para la primaria completa y un $26 \%$ con primaria incompleta y analfabetismo.

En relación con la ocupación, las mujeres desempeñan roles de género tradicionales, casi todas estaban dedicadas por completo al cuidado de los(as) hijos(as) y a la realización de las tareas domésticas. Por otro lado, los hombres eran los principales proveedores del sustento económico de las familias, a través del trabajo como pescadores, constructores y artesanos. Los hogares muestran un alto grado de vulnerabilidad económica, especialmente, porque dependen de un solo ingreso, en su mayoría estos provienen del trabajo por cuenta propia y además el trabajo escasea por temporadas, por ejemplo, para los peones de finca la época de verano presenta poca demanda de trabajo, para los pescadores, los fuertes vientos y las mareas altas impiden la captura de producto.

\section{Ingresos de los hogares}

Aunque este estudio tiene un enfoque donde en enfatiza lo cualitativo, se considera importante complementar el mismo con algunos datos cuantitativos de gran importancia sobre las dinámicas socioculturales que estos grupos humanos tienen en su diario vivir. Tal es el caso de la información sobre los ingresos monetarios. 
Cuantificación de los Ingresos: de acuerdo con sus ingresos, los hogares con una condición económica más desfavorable se encontraron en Palenque Margarita en donde $67 \%$ de ellos vive en pobreza extrema, de seguido la Isla de Chira con $60 \%$ y Acosta que presenta un escalafón más bajo, con $45 \%$. Se considera que en las tres comunidades el nivel de pobreza extrema es realmente alto, aun percibiendo apoyo económico estatal a través de sus diferentes programas sociales.

La tabla $\mathrm{N}^{\circ} 1$, muestra el ingreso per cápita promedio por comunidad de dos formas, primeramente se consideran los ingresos producto del trabajo por cuenta propia, asalariado, las pensiones alimentarias y las transferencias por parte del Estado (pensiones y becas de estudio). En segundo término, se hace una estimación de los ingresos excluyendo los apoyos económicos estatales.

Tabla $\mathrm{N}^{\circ} 1$

Ingreso per cápita de los hogares, estudiados con y sin ayuda estatal, oct. 2014 (Colones)

\begin{tabular}{lcc}
\hline Lugar & $\begin{array}{c}\text { Ingreso per cápita con apoyo } \\
\text { estatal }\end{array}$ & $\begin{array}{c}\text { Ingreso per cápita sin } \\
\text { apoyo estatal }\end{array}$ \\
\hline Acosta & $\mathbb{C} 53.665$ & $\mathbb{C} 42.020$ \\
Isla de Chira & $\mathbb{C} 36.805$ & $\mathbb{C} 28.148$ \\
P. Margarita & $\mathbb{C} 40.156$ & $\mathbb{C} 23.217$ \\
\hline
\end{tabular}

Fuente: Elaboración propia con datos de la encuesta.

Como podrá observarse, sin apoyo estatal los ingresos per cápita se ven seriamente disminuidos en $\mathbb{\$} 12.414$ en promedio (\$23), pero a nivel de comunidad, los ingresos de los hogares de Palenque Margarita son los más afectados ya que muestran la mayor disminución. La ausencia del apoyo económico estatal no solo disminuiría los ingresos de los hogares en un $28 \%$ en promedio, lo que equivale a $\$ 11.960$ (\$22), sino que además incidiría en aumentar la condición de pobreza de los hogares, pues un $26 \%$ de ellos se mantendría en pobreza, mientras que el $74 \%$ pasaría a estar en pobreza extrema.

Es claro que el papel del Estado como paliativo a la pobreza es fundamental, pues establece una especie de anclaje que la sostiene evitando un 
deterioro mayor en las condiciones de vida de estas familias. Según el XX Informe del Estado de la Nación, "la red de programas sociales que tiene el país funciona como una malla de contención de la pobreza, de no existir estos programas la pobreza en el país se duplicaría". (Rivera, 2014).

Lo anterior se confirma cuando según estimaciones realizadas por el Programa Estado de la Nación del año 2014, señala que si al ingreso de los hogares se le suman las transferencias que reciben los beneficiarios del Instituto Mixto de Ayuda Social (IMAS) y el Fondo Nacional de Becas, así como las pensiones del Régimen no Contributivo, el efecto global de estos programas es una reducción de aproximadamente dos puntos porcentuales en la pobreza. (Programa Estado de la Nación, 2014).

Poco más de la mitad de los hogares presentan la particularidad de tener ingresos inestables, pues la mayoría provienen de la realización de trabajos por cuenta propia, como peones de fincas, pescadores, constructores y además dependen de un solo ingreso, aportado por los hombres. Los hogares mono parentales a cargo de una mujer, también se ven afectados por inestabilidad, que tiene lugar cuando las pensiones alimentarias son depositadas o dadas a destiempo o no son otorgadas del todo, lo que ocurre con alguna frecuencia. Por el contrario, la estabilidad de los ingresos se encuentra en los hogares que dependen del trabajo asalariado o que reciben pensiones por parte del Estado.

En cuanto a la administración del dinero, en los hogares nucleares tanto en Acosta como en Isla de Chira son principalmente los hombres quienes lo hacen. A diferencia de Palenque Margarita donde la mayoría de las mujeres manifestaron ser ellas quienes deciden cómo y en qué se gasta y priorizar la compra de alimentación y el pago de servicios de electricidad y agua. En los hogares estudiados, las mujeres demuestran tener una visión más integral de las necesidades familiares y esto les permite hacer una mejor administración de los recursos económicos. Además los papeles o funciones de género socialmente asignados a las mujeres tales como los oficios domésticos, la crianza y cuidado de los (as) hijos(as), hacen que tengan conocimientos de las diferentes necesidades de sus familias. (Guerin, 2010).

Composición de los ingresos: en los hogares de las tres comunidades, los ingresos provienen de diversas fuentes las cuales se detallan 
a continuación. En poco más de la mitad (58\%) de los hogares, son los hombres quienes aportan el ingreso familiar, con un monto promedio de $\$ 146.821$, el equivalente a $\$ 267$. El aporte monetario de las mujeres solo se encontró en pocos hogares (16\%). Esta escaza participación se explica por varios motivos, uno de ellos es la existencia o prevalencia de la división sexual del trabajo que impera en todas estas familias, en donde las mujeres se encontraban realizando trabajo doméstico y atención de sus hijos(as), que en buena parte eran menores de edad. Habría que agregar que la mayoría de ellas no había tenido experiencias laborales y manifestaron no tener interés en trabajar fuera de su casa, pero habría que agregar que las comunidades presentan muy pocas o nulas opciones de trabajo femenino.

En este punto es importante señalar que no obstante que las mujeres no hacen un aporte monetario a sus hogares, la realización de las tareas domésticas y el cuidado de hijos(as) y de otros integrantes del hogar constituye un valioso aporte al mismo, pues en el seno de la familia se reconstituyen las acciones de reproducción de la fuerza de trabajo. Autoras como Benería (1998) y Carrasco (2006), al igual que otras economistas feministas, han puesto en evidencia la importancia social y económica que reviste estas funciones para la reproducción social de las familias, del conjunto de la sociedad y del sistema económico.

Las becas de estudio de los dos programas estatales, Avancemos y el Fondo Nacional de Becas (Fonabe), están presentes en un $60 \%$ de los hogares, con un monto promedio de $\mathbb{\$ 3} 3.000$ (\$60).

Desde el criterio de la investigadora, todas las becas fueron bien asignadas, pues las familias reúnen el principal requisito que es -estar viviendo en situación de pobreza-. No hay duda que sin este tipo de apoyo es muy posible que las familias no tengan las condiciones materiales para que sus hijos(as) puedan estudiar y con ello se estaría reproduciendo el ciclo de la pobreza o traspasando la pobreza a otra generación. En muchas ocasiones estos dineros pasan a formar parte del ingreso familiar ya que se destinan a la compra de alimentación y a sufragar otros gastos de la familia.

Por otra parte, las pensiones del Régimen no Contributivo (RNC) aparecen en un $14 \%$ de los hogares. El monto promedio de esta pensión era de $\$ 70.000$ (\$127). Las pensiones alimentarias son otra fuente de ingreso 
particularmente en los hogares a cargo de mujeres, $20 \%$ del total de los hogares recibía estas pensiones. Los subsidios del IMAS, llegaban a una tercera parte de los hogares a través de los dos programas Manos a la

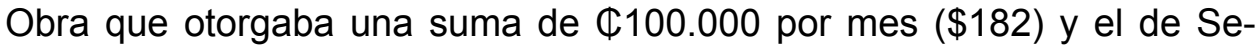
guridad Alimentaria, destinado a jefas de hogar y cuya asignación era de $\$ 50.000$ (\$91).

Es importante resaltar que, un $82 \%$ de los hogares de las tres comunidades se encontraba recibiendo algún tipo de apoyo económico estatal, a través de los diferentes programas sociales, como los antes mencionados. Los hogares también perciben otras transferencias no monetarias las cuales se detallan más adelante.

\section{Composición del gasto en los hogares}

Los gastos que presentan estas familias son: la compra de alimentación, el pago de servicios, electricidad, agua, teléfono y cable, alquiler de casa, gastos en educación y pago de deudas. A continuación se hace una descripción de cada uno de ellos.

Alimentación: la alimentación por lo general consiste en arroz, frijoles, café, azúcar, aceite o manteca, pasta o fideos, huevos, algunas verduras y hortalizas. Este gasto consume en promedio la mitad del ingreso familiar en los hogares de las tres comunidades.

La mayoría de las familias están en condición de poder hacer los tres tiempos de alimentación, es decir, desayuno, almuerzo y cena, solo que nueve de cincuenta familias no estaba en capacidad de hacerlo y las mujeres manifestaron que en algunas ocasiones "pasan hambre". Esta situación afectaba más a las familias de Palenque Margarita, donde una tercera parte de las mujeres manifestaron que en algunas ocasiones no disponían de alimentos para desayunar, almorzar o cenar.

La alimentación de las familias se ve complementada con el consumo de productos de la zona, como son el pescado y camarones, en el caso de Isla de Chira, de aguacates, flores de itabo, naranjas y otras frutas que se producen en Acosta y en Palenque Margarita, los plátanos verdes y maduros, bananos, pejibayes y yuca. Esto es importante porque cuando las personas comen solamente arroz y frijoles les genera un "sentimiento de 
estar comiendo pobremente", pero si le agrega algo más, este sentimiento tiende a diluirse. Además estos productos mejoran la nutrición de las personas, pues las frutas, verduras y hortalizas aportan vitaminas y minerales.

Es importante mencionar que un $72 \%$ las familias también se ven beneficiadas por la existencia del comedor escolar, en donde sus hijos e hijas de primaria y secundaria reciben alimentación de este programa a razón de dos estudiantes promedio por hogar. Por otro lado, los servicios del Centro de Educación y Nutrición y de Centros Infantiles de Atención Integral (CEN-CINAI), casi llegaban a la mitad (48\%) de los hogares a través de alimentación y bolsas de leche para los más pequeños(as) y para las mujeres. Hay que recordar que estos centros de atención, son dependencias del Ministerio de Salud que tienen como función el contribuir a mejorar el estado nutricional de la niñez y de mujeres en estado de embarazo y post embarazo. Se tiene que buena parte $(77 \%)$ de los hogares recibía beneficios de alguno de los tres programas estatales antes mencionados.

Pago de servicios: el pago de los servicios de electricidad y agua, es el segundo gasto que efectúan las familias una vez que han comprado la alimentación. Este pago representaba en promedio un 15\% del ingreso familiar, siendo más oneroso el de la electricidad, y que en algunas ocasiones se les hace difícil reunir el dinero para pagarlo y por ello sufren la suspensión o "corta" de tan importante servicio.

Una tercera parte de las familias disponían de televisión por cable, este gasto representaba en promedio un $8 \%$ del ingreso familiar. De primer momento, podría leerse -desde afuera de la comunidad- como un gasto superfluo, relativamente costoso para los presupuestos de esas familias, sin embargo, este servicio se convierte en uno de los principales medios de acceder a la "televisión nacional", cuando la recepción de la señal de aire es deficiente en el lugar donde residen. Estas familias, forman parte de la estadística nacional, que según la Encuesta Nacional de Hogares para el año 2014 , un $62 \%$ de las viviendas del país contaban con cable de televisión. (Leitón, 2015).

El acceso a este tipo de servicio facilita generar un sentimiento de inclusión social, de pertenencia cultural a la Nación, al poder disponer de la información al igual que otros grupos sociales y que se concreta mediante programas noticiosos, deportivos y en general de entretenimiento. A lo 
anterior se aúna el contacto con la cultura global que brinda la tecnología en estos tiempos.

Gastos en educación: los hogares presentan gastos por concepto de lo que podría llamarse "gastos en educación", este aparece en una tercera parte de estos. Por lo general, este gasto incluye dinero que se da a los(as) estudiantes de escuela y colegio para la compra de alguna merienda o de algún material escolar. Este representa en promedio un $9 \%$ del ingreso familiar. Por lo general, este dinero es tomado de las becas que reciben los estudiantes de los programas antes citados. Las familias no incurren en gasto por concepto de transporte ya que en algunos casos tanto la escuela como el colegio son lugares accesibles y se puede llegar caminando o en bicicleta, en otros, el Ministerio de Educación, asume el pago de este servicio. Este es un mecanismo que al igual que la becas busca generar las condiciones para que los y las estudiantes puedan asistir a escuelas y colegios, pues la educación es la apuesta para combatir la perpetuación de la pobreza.

Para el inicio del período escolar, las familias prácticamente registran poco gasto, sencillamente porque no tienen capacidad para comprar estos enseres. Por lo general, se reutiliza la ropa del año saliente, o tratan de obtenerla de otras personas de la comunidad. Algunas pocas familias, van guardando algo de dinero de los ingresos, de las becas, o de los subsidios del IMAS para poder adquirir ropa y útiles escolares. La mayor dificultad está en los zapatos, no solo porque son caros sino porque casi nadie los regala.

Pago de deudas: al momento de visitar los hogares para recoger la información, un porcentaje importante de las familias se encontraban pagando deudas (66\%). Este gasto constituía en promedio un $14 \%$ del total del ingreso. Para efectos de este trabajo, solo se tomó en cuenta el monto pagado mensualmente de forma fija. Las deudas se derivan de la compra de electrodomésticos como: lavadoras, televisores, cocinas, computadoras, ventiladores, tabletas, teléfonos celulares, bicicletas y motocicletas. Con respecto a estos bienes se pudo observar un uso diferenciado, por ejemplo, las mujeres utilizan aquellos artefactos que contribuyen a facilitar la realización del trabajo doméstico. Las tabletas, computadoras y teléfonos son mayormente solicitados por los(as) jóvenes y por estudiantes para realizar sus trabajos. 
En Acosta e Isla de Chira se encontraron deudas por concepto de la compra de motos y bicicletas, ambos son medios de transporte importantes y necesarios para hacer "mandados" $y$ para desplazarse al trabajo, escuela o colegio. También se encontraron deudas con "polacos" ${ }^{5}$ por concepto de compra de ropa, zapatos, pero también de comida, cuando esta se ha comprado "fiada" o a crédito en pulperías del lugar.

Las familias recurren a la compra a crédito como una forma de poder hacerse de los bienes necesarios que no pueden comprar de contado. En el país la venta a crédito está muy desarrollada como una forma de incentivar el consumo, por lo general, las tiendas y almacenes ofrecen condiciones de pago para familias de poco poder adquisitivo como las de este estudio. Las mujeres mencionaron también que suelen atrasarse en el pago de las mensualidades del crédito, ya que cuando hay poco dinero, priorizan la compra de alimentación y el pago de los servicios. Datos de un estudio realizado, revelan que un $22 \%$ de los hogares costarricenses se encontraban pagando deudas de electrodomésticos, tal y como lo hacían las familias estudiadas en este trabajo. (Avendaño, 2016).

Con las dificultades económicas que viven estos grupos, la compra a crédito-aunque más onerosa- es el medio para poder adquirir algunos bienes que les resultan prioritarios. Incluso para la alimentación se recurre a créditos en negocios locales que tienen una política de "fiado" que logra alivianar las carencias económicas coyunturales.

Sobre la ropa: tres cuartas partes de las familias, hacen uso de ropa regalada por familiares y amistades, pues no están en capacidad de comprar, a lo sumo usada "tipo ropa americana". Salvo excepciones, como un cumpleaños, día de la madre o época navideña, se puede adquirir ropa nueva. Las familias tampoco pueden comprar juguetes y las iglesias evangélicas y católicas colaboración con algunos regalos para niños y niñas especialmente en navidad, sobre todo en Isla de Chira y P. Margarita.

\section{Vivienda, servicios básicos y equipamiento}

Es importante mencionar que la mayor parte de las familias (90\%) disponían de una vivienda propia, que pese al mal estado físico, las personas

4 En Costa Rica hacer un "mandado", es ir a realizar un trámite, una diligencia o una compra.

5 "Polaco", es aquella persona que vende mercadería diversa, a crédito en las comunidades. 
logran vivir en ellas con un nivel respetable de bienestar y además pueden dormir con tranquilidad, privacidad y comodidad. El papel del Instituto Mixto de Ayuda Social (IMAS) ha sido determinante en la dotación de vivienda, ya sea otorgando dinero para construir la casa, entregando la casa construida o brindando ayudas económicas para la ampliación de las mismas.

El tener resuelto el lugar donde vivir tiene varios significados para la mayoría de las familias; por un lado, no incurrir en gastos por concepto de alquiler y con ello aliviar el presupuesto familiar, el poder vivir con la tranquilidad de saber que se tiene una casa propia o "relativamente propia" lo que da lugar a asentarse en un sitio y construir arraigo. Por otro lado, en el caso específico de la mujer, el saberse dueña o propietaria de una vivienda genera un sentimiento que lleva a no "sentirse pobre", pues ellas definieron la pobreza como la situación que viven personas que no tienen "ni techo ni comida".

Las viviendas de las tres comunidades cuentan con servicios básicos de electricidad, agua potable y sanitario. La disposición de alumbrado y energía de manera permanente no solo facilita la realización de las tareas domésticas a cargo de las mujeres, sino además el estudio, la información e interacción y bienestar para el grupo familiar. Sobre el agua es importante señalar dos aspectos, primero que su potabilidad contribuye a evitar problemas de salud, y segundo, la ubicación dentro del domicilio, que facilita el trabajo doméstico y evita la labor de acarreo, con las consecuencias perjudiciales para la salud de las personas que lo realizan.

Habría que agregar que buena parte de las viviendas disponían de un equipamiento básico, que consistía en televisor, lavadora, refrigeradora y olla arrocera. Todo esto no solo facilita las tareas hogareñas realizadas por las mujeres, sino que además provee de bienestar y calidad de vida al conjunto de la familia. A estos enseres habría que agregar que, en la mayoría de los hogares, disponían de teléfonos celulares o residenciales, lo que permite un nivel de comunicación global, pues muchos de ellos tienen conexión con internet.

\section{Acceso a servicios de salud}

La mayor parte de las familias cuentan con seguro social, la mayoría por parte del Estado. Este garantiza el acceso a la atención en salud a aquellas 
personas que por condición económica no pueden cotizar para algún régimen. Esta modalidad de aseguramiento, es un mecanismo inclusivo y solidario, pues las personas tienen derecho a una atención primaria en salud, lo mismo que a especialidades médicas e intervenciones quirúrgicas cubiertas por el seguro.

También es accesible el servicio de atención primaria en salud, brindada por los Equipos Básicos de Atención Integral en Salud (EBAIS) que se encuentran ubicados en las mismas comunidades o a una distancia relativamente corta. Las familias de Isla de Chira, tienen la desventaja de que la condición de isla implica una limitación en el acceso a la salud, pues muchas veces no disponen de los recursos económicos para pagar el transporte en lancha para asistir a una cita médica.

Otro aspecto importante de mencionar es que la precariedad e inestabilidad de los ingresos impacta o afecta negativamente la salud de las mujeres, pues cuando el dinero no alcanza para atender las necesidades familiares, la intranquilidad les produce dolor de cabeza, aumento en la presión arterial y en el nivel de azúcar en la sangre, insomnio y deseos de llorar, con el agravante de que esto sucede con relativa frecuencia.

Adicionalmente, se puede mencionar también otro elemento que incide de manera negativa en la salud de las mujeres, tiene que ver con una cultura rural que considera que la alimentación cocinada con leña tiene mejor sabor, junto con la preocupación por la reducción de la tarifa eléctrica, lleva a las mujeres a cocinar con fogón de leña. La exposición al humo de manera frecuente ocasiona, problemas respiratorios y aumenta las posibilidades de padecer cáncer de pulmón a las mujeres y a otros integrantes de la familia.

Por otro lado, es importante rescatar la valoración positiva que hicieron las mujeres del entorno donde viven, aprecian habitar en lugares rodeados de naturaleza silvestre, con aire limpio, con espacios amplios y accesibles que permiten disfrutar de paz, tranquilidad y seguridad. Reconocen la relación amigable que existe entre vecinos, valoran que los niños(as) puedan desplazarse solos a las escuela y que disponen de espacios como plazas y patios para la recreación. 


\section{DISCUSIÓN}

Pobreza multidimensional: la pobreza, abordada de manera multidimensional permite hacer visibles una serie de aspectos que inciden en la calidad de vida, que solamente el ingreso no lo hace posible. Las familias pobres de esta investigación son un claro ejemplo, pues en ellas se puede observar la existencia de pobreza con alimentación básica, pobreza con tenencia de vivienda, pobreza con acceso a los servicios de salud. En este sentido, el estudio de la pobreza, desde la multidimensionalidad y de manera cualitativa, es un acierto, ya que permitió hacer una valoración de la misma de manera más integral. De igual forma, evidenció el impacto y la importancia que tienen los programas sociales y su incidencia en la pobreza de estas familias.

La pobreza como categoría: la pobreza suele ser una categoría que además de permitir ubicar a las personas de acuerdo con sus ingresos, tiende a "marcar" a quienes están en esa condición, introyectándolas a un determinismo de su visión de mundo. No obstante, se determinó que no es el caso de las mujeres del estudio, quienes desde su subjetividad perciben sus condiciones de vida- que trasciende lo económico- sin el estigma de ser pobres. Las mujeres, no solo no se asumieron como tales, sino que además tienen una visión de futuro optimista y positiva, lo que contribuye en un buen estado físico y emocional. Es posible que en esta percepción intervengan factores diversos, pero sin duda, el Estado y sus políticas sociales, les permiten tener la esperanza de un futuro con mejores condiciones de vida.

Escasez de trabajo: las tres comunidades presentan escasas opciones laborales para los hombres y para las mujeres; esto hace que las familias vivan una condición de pobreza por falta de ingreso, que las hace altamente dependientes de las ayudas o apoyos estatales y posiblemente lo sigan siendo al no vislumbrarse cambios en la estructura productiva de los lugares rurales donde residen. Unido a lo anterior, hay que señalar que el sector agrícola costarricense ha venido mostrado un debilitamiento que se evidencia en una menor presencia o contribución en el Producto Interno Bruto (PIB).

Trabajo por cuenta propia: el trabajo por cuenta propia que predomina en los hombres, esposos o compañeros de las mujeres, genera una condición 
de vulnerabilidad socio económica que se expresa en la inestabilidad en los ingresos, carencia de garantías laborales tales como el pago de seguro social, incapacidad por motivos de salud, vacaciones, aguinaldo y cotización para algún régimen de pensión. Esto impacta de manera negativa al conjunto de familias pues no se tienen aseguradas o garantizadas ciertas condiciones económicas que provean una mejor calidad de vida y bienestar en el presente y que puede comprometer su futuro.

Origen de los ingresos: los ingresos de las familias presentan la particularidad de que provienen de diversas fuentes u orígenes: de salarios, producto del trabajo de los hombres, y en menor grado de de hijos(as) y de otros integrantes, de pensiones alimentarias, de pensiones del Régimen No Contributivo (RNC) y de los diferentes programas sociales estatales. En la mayoría de los hogares nucleares, son los hombres quienes aportan el ingreso para mantener a la familia. Por el contrario, son muy pocas mujeres y en forma ocasional las que hacían un aporte monetario. Su contribución más bien está en la realización del trabajo doméstico, tan importante para la reproducción del núcleo familiar.

Hogares con alta dependencia:en los hogares hay alta presencia de personas jóvenes, prácticamente la mitad son hijos(as) y algunos nietos, tienen menos de dieciocho años de edad. Esto muestra un alto nivel de dependencia con respecto de sus padres y madres en proveerles alimentación, educación, vestimenta y cuidados. De ahí, la responsabilidad que tienen estos de generar los recursos materiales y no materiales en condiciones adversas de oportunidades de trabajo, para sacar adelante la tareas de criar y cuidar a estos hijos(as) y llevarlos(as) hasta la adultez para que adquieran su independencia.

Estado aporta transferencias monetarias: el Estado contribuye en buena medida a mejorar y aumentar los ingresos familiares, pues estas "ayudas" estaban llegando a un porcentaje importante de las familias. Estas se materializaban a través de las becas de estudio de los Programas Avancemos y del Fondo Nacional de Becas, de subsidios del IMAS (Programas Manos a la Obra y Seguridad Alimentaria), de pensiones y del Régimen no Contributivo. Estos apoyos son fundamentales para la subsistencia de las familias, pues sin estos su condición de pobreza sería más severa, amenazando incluso la reproducción más elemental de sus condiciones de vida, pues los ingresos disminuirían de manera importante como ya se demostró. 
Estado aporta otras transferencias no monetarias:los presupuestos de las familias también se ven beneficiados por otras transferencias otorgadas por el Estado. El primero de ellos, sería el acceso al comedor escolar, el segundo, son los servicios del CEN-CINAI, estos servicios beneficiaban a un importante porcentaje de familias y de igual manera, logran disminuir la presión sobre el presupuesto, pero sobre todo aportan significativamente en la reproducción del grupo familiar. Sin lugar a dudas, sin la recepción de estos importantes servicios, la salud, la nutrición, y en general el desarrollo físico y emocional de las personas se vería afectado.

Dinero insuficiente para atender necesidades familiares: no obstante y el apoyo recibido por parte del Estado, los ingresos de las familias son insuficientes para cubrir las necesidades del conjunto de sus integrantes. El dinero alcanza para adquirir una canasta básica de alimentos, para pagar los servicios y el alquiler de casa. El dinero no es tan elástico como para poder adquirir una mayor cantidad y variedad de alimentos como es lo deseado, tampoco para comprar zapatos, ropa, medicamentos, para hacerle reparaciones a la vivienda y otros. El nivel de satisfacción de las necesidades es mínimo, como mencionan algunas mujeres "es apenas para ir sobreviviendo".

Gastos básicos: El conjunto de los gastos elementales consume casi la totalidad del ingreso. La compra de la alimentación y el pago de los servicios son los gastos prioritarios para una familia, la primera, prácticamente consume la mitad del ingreso y consta de una canasta muy básica, pues la lista incluye un mínimo de enseres necesarios para garantizarse una sobrevivencia. Es muy poco, el dinero que "sobra", pues los gastos se calculan sobre generalidades, pero casi siempre surgen otras necesidades e imprevistos que no se consideran.

Endeudamiento como medio de sobrevivencia: la mayor parte de las familias recurren a la compra a crédito como un medio de adquirir los bienes cuando los ingresos no lo permiten. El sistema crediticio en el país facilita que este tipo de familias puedan ser consumidoras de enseres que logran dar un sentido de inclusión e igualdad social en tiempos donde los sujetos se construyen a partir de la tenencia y disfrute de bienes materiales. El endeudamiento constituye una estrategia de sobrevivencia a fin de hacerse de una serie de bienes que las familias consideran como importantes. El poder tener acceso a estos contribuye también a no sentirse 
pobres, pues tienen artículos que el conjunto de la sociedad costarricense posee. Sentirse incluida en una sociedad con la mediación de artefactos es de vital importancia en la autoestima de las personas.

Pobreza y subjetividad:la manera de cómo sienten y viven su condición socioeconómica las personas que se clasifican como "pobres", revela como la subjetividad en la percepción de su realización a la luz de sus escasos y limitados recursos, no generan en ellos una desesperanza, amargura o rendición frente a la adversidad. Más bien, su propia representación social les da en el día a día una percepción de oportunidad, que usualmente no coincide con las condiciones objetivas en que desarrollan sus vidas.

\section{REFERENCIAS BIBLIOGRÁFICAS}

Alkire, S. \& Santos, M.E. (2010). Acute Multidimensional Poverty: A New Index for Developing Countries. OPHI Working Paper $\mathrm{N}^{\circ}$ 38. Oxford Poverty \& Human Development Initiative (OPHI). Oxford Department of International Development Queen Elizabeth House (QEH), University of Oxford. Recuperado de https://www.ophi.org.uk/wp-content/ uploads/ophi-wp38.pdf

Avendaño, M. (27 de mayo del 2016). 22\% de los hogares debe electrodomésticos. La Nación, pág. 20 A.

Benería, L. (1998). La mujer y el género en la economía: un panorama general. En Paloma de Villota (Ed). Economía y Género. Macroeconomía, Política Fiscal y Liberalización, Análisis de su impacto sobre las mujeres. Barcelona: Icaria Editorial S.A.

Bravo, R. (1998). Pobreza por razones de género. Precisando conceptos. Género y pobreza: nuevas dimensiones. Isis Internacional. Ediciones de las Mujeres $\mathrm{N}^{\circ} 26$.

Carrasco Cristina. (2006). La economía feminista: una apuesta por otra economía. En: Vara, M. J. (coord.). Estudios sobre género y economía. Akal, Madrid.

Castro, A. M. (2015). Fichero Cantonal 2016. Instituto de Formación y Estudios en Democracia. Tribunal Supremo de Elecciones. San José, Costa Rica. 
CEPAL-UNIFEM. (2004). Entender la pobreza desde la perspectiva de género. Serie Mujer y Desarrollo $N^{\circ} 52$. Unidad Mujer y Desarrollo. Santiago de Chile.

Chant, S. (2003). Nuevas Contribuciones al análisis de la pobreza: desafíos metodológicos y conceptuales para entender la pobreza desde una perspectiva de género. Serie Mujer y Desarrollo $N^{\circ} 47$. Unidad Mujer y Desarrollo, CEPAL. Santiago, Chile.

Instituto Nacional de Estadística y Censos [INEC]. (2011). Encuesta Nacional de Hogares. Cifras Básicas sobre fuerza de trabajo, pobreza e ingresos. INEC, San José, Costa Rica.

Instituto Nacional de Estadística y Censos [INEC]. (2016). Recuperado de: www.inec.go.cr/sites/default/files/documetos-biblioteca-virtual/repoblacpanorama2016.pdf

Ministerio de Planificación Nacional y Política Económica [Mideplan]. (2013). Área de Análisis del Desarrollo. Índice de Desarrollo Social 2013. MIDEPLAN, San José, Costa Rica.

Comisión Económica para América Latina [CEPAL] (2013). La medición multidimensional de la pobreza. Duodécima reunión del Comité Ejecutivo de la Conferencia Estadística de las Américas de la Comisión Económica para América Latina y el Caribe. Pucón (Chile), 24 a 26 abril de 2013.

Guerin, I. (2010). Las mujeres pobres y su dinero: entre la supervivencia cotidiana, la vida privada, las obligaciones familiares y las normas sociales. Estudios de género La Ventana, 32 (4), 7-51.

Kabeer, N. (1998). Más allá de la Línea de Pobreza: medición de la pobreza y medidas que empobrecen. Realidades trastocadas. Las jerarquías de género en el pensamiento del desarrollo. Programa Universitario de Estudios de Género. Universidad Nacional Autónoma de México. Paidós, México.

Leitón, P. (31 de octubre del 2015). Internet se encuentra en seis de cada diez hogares del país. La Nación, pág. 22A. 
Programa Estado de la Nación en Desarrollo Humano Sostenible, Costa Rica. (2014). Décimo sexto Informe Estado de la Nación en DesarroIlo Humano Sostenible. Programa Estado de la Nación en Desarrollo Humano Sostenible. San José, Costa Rica.

Rivera, E. (12 de noviembre del 2014). La pobreza tiene remedio. Semanario Universidad, pág.4-5.

Sen, A. (1999). Desarrollo y bienestar. Editorial Planeta, Barcelona, España. 
